\title{
DIGITAL IMAGE PROCESSING AND CCD IMAGING AT GOTHARD ASTROPHYSICAL OBSERVATORY
}

\author{
I. JANKOVICS, J. KOVÁCS and I.J. VINCZE \\ Gothard Astrophysical Observatory of Lorand Eötvös University \\ Szent Imre Herceg u. 112 \\ H-9707 Szombathely \\ Hungary
}

\section{Introduction}

The Gothard Astrophysical Observatory of Loránd Eötvős University was founded in 1881 by Eugene von Gothard, the first astronomer in the world who took a photo of the central star of Ring nebula. After nearly 80 years interregnum, the observatory was revitalized by Eötvös University in 1992 and its equipment is used both for education and for research.

The main topic at Gothard Astrophysical Observatory is the investigation of emission line stars using high dispersion echelle spectrograms.

The work on digital image processing was begun two years ago. Our intention was to set up a world wide astrophysical observatory with the most advanced technical background.

Gothard Astrophysical Observatory is equipped with a Sun workstation based computer network which is an excellent tool for data and image processing. The Observatory is a node of the wide area computer network.

\section{Scientific Activity}

Our main scientific goal is the investigation of the short term variations of early type emission line stars (Be stars and some supergiant stars) using photoelectric photometry and high resolution spectroscopy.

Now we are starting the study of the long term brightness variations of flare stars using the Hungarian plate archives on Pleiades.

\section{Technical and Computational Background}

An extensive development was begun two years ago. The 600/7500 Cassegrain telescope of the observatory was equipped with a modern, fully automated and computer controlled stellar photometer armed with Johnson UBVR, Strömgen ubvy and $H \beta$ narrow and wide band filters for photoelectric observations and a CCD camera was obtained for direct imaging.

At the current stage of development, seven computers are organised into a local area network, which is connected with the wide area network via INTERNET protocol. The heart of the system 


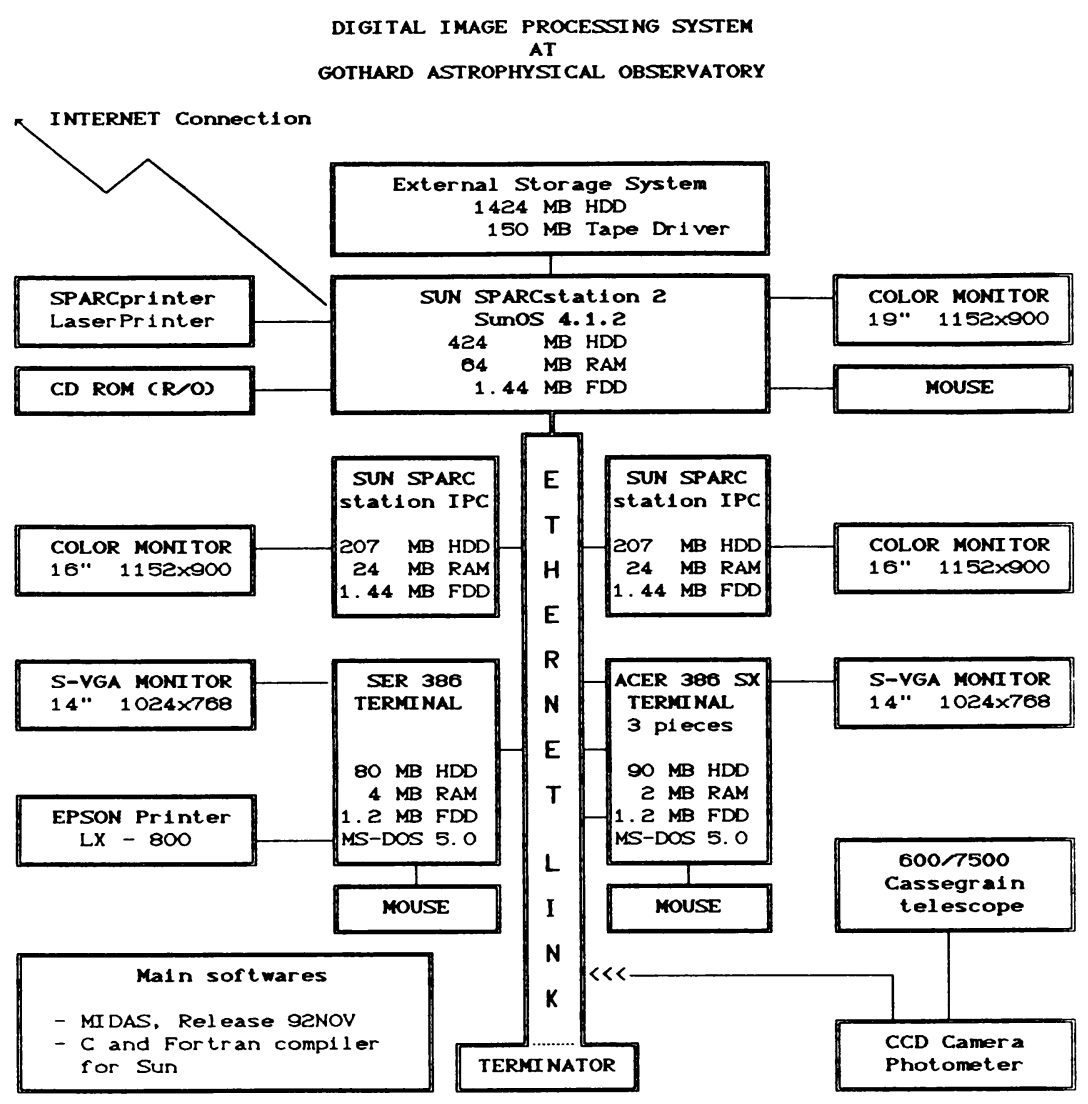

is a Sun cluster which contains one Sun SPARCstation 2 (as a server), and two Sun SPARCstation IPCs running under SunOS 4.1.2 operating system. These machines have 64 and 24 megabytes of memory, respectively. The total hard disk capacity is more than $2 \mathrm{~GB}$. There are four additional PCs linked to the server which are able to work as $X$ terminals. The main software we use for data reduction and analysis is the Munich Image Data Analysis System (MIDAS).

\section{Future Plans}

It is planned to construct a modified version of the portable echelle spectrograph of the Landessternwarte Heidelberg which will be an ideal instrument for long-term spectroscopic monitoring programs at the $0.6 \mathrm{~m}$ telescope of the Gothard Astrophysical Observatory. This can produce a large set of data in short time scales and can open numerous opportunities of joint science research projects with European astronomical institutions.

The technical development program is driven by opportunities to develop new areas of astronomy requiring continuous and long term monitoring both photometrically and spectroscopically. There are considerable scientific returns to be had by using this equipment in parallel with satellite and other waveband observations. 